\title{
FIRST 1000 DAYS: USING 'EMERGENGE' TO TAKE SOCIAL INNOVATION TO SCALE
}

\section{BY KERRY ARABENA, LUELLA MONSON-WILBRAHAM, ELLE MCLACHLAN, ALANA MARSH AND MARION CALLOPE}

\section{ABSTRACT}

Enhancing health and wellbeing outcomes for infants is at the forefront of global Indigenous social work practice. An evidence informed international 1,000 Days movement, which focuses on nutrition during the period from conception to a child's second birthday, has been expanded by the First 1000 Days Australia model to include Indigenous holistic and cultural perspectives to support First Nation infants and their families. Developed and delivered by Indigenous peoples in Australia, Indonesia and Norway, this paper explores how the model works with the ecological theory of emergence to support parents and carers enact cultural protections for Indigenous children during the early formation of their families. 


\section{INTRODUCTION}

Aboriginal and Torres Strait Islanders are the two distinct Indigenous populations in Australia, totalling approximately one million people or 3 per cent of the total Australian population. Australian Aboriginal and Torres Strait Islander social workers are acutely aware that, since the colonisation of Australia in 1788, our people are some of the most disadvantaged in Australian society. Indigenous social workers are drawn to the field to create conditions in which our people are empowered to 'live a good life'. What differentiates non-Indigenous from Indigenous social workers is a drive to attain equality and to promote our culture as the protective factor for our families. In Australia, Social Work practice has become synonymous with tertiary levelled interventions, which sees many of our children removed from families because of the crisis driven nature of the work. To move from tertiary levelled interventions to preventative ones are critical; but difficult to achieve in service delivery systems that are geared toward child removal, not child protection.

First 1000 Days Australia is a strategy that is conceived and led by Indigenous people, that mobilizes community and Indigenous resources in a time specific intervention from pre conception until a child's second birthday. These one thousand days represents a time of vulnerability for the developing infant; and a period of enhanced opportunity to use culture led practices to promote family wellbeing. This is because every mother and father have a story to tell about the beginnings of their child's life. Many of them are joyful, some are heartbreaking and all of them important. Cultural determinants of health and wellbeing in an Australian context include six broad domains: connection to country; cultural beliefs and knowledge; language; family, kinship and community; expression and cultural continuity and self-determination and leadership (Salmon et. al, 2018). These cultural domains are enacted in First 1000 Days Australia through family engagement, workforce development, systemic reform, policy and programming, advocacy, knowledge exchange, Indigenous governance and leadership strategies as well as economic empowerment through the growth and support of Indigenous businesses.

This article focuses on the development and delivery of time-specific interventions that engage parents, extended families and their children through regional initiatives, across different sectors and countries. We explain why the 
'lifecycle of emergence' is a core theoretical underpinning of our efforts in this, and present case studies that demonstrate how we work with local and regional areas to develop our initiatives. Finally, we highlight the lessons we have learned that garner and support success in the implementation of regional social emergence strategies, specifically those targeting Indigenous peoples.

\section{PRIVILEGING FAMILIES IN THE FIRST 1000 DAYS}

A council made up of Aboriginal and Torres Strait Islander Elders, researchers, community members, front line workers and policy makers ensures First 1000 Days Australia is led by Aboriginal and Torres Strait Islander people and employs Indigenous methods of knowledge generation and family engagement. We support parents in identifying and achieving their aspirations, so they can address their children's needs from pre-conception to two years of age, thereby laying the best foundation for their future health and wellbeing. We privilege families as the primary and preferred site for developing and protecting culture and identity in Aboriginal and Torres Strait Islander children and our scope of work is used to support families, organisations and communities to better prepare for and respond to the needs of their children. Founded on partnerships to promote collective impact, the model takes a multigenerational view of the family and what these generations can offer to the family to grow healthy children.

Aboriginal and Torres Strait Islander Australians who choose social work as a calling, acknowledge that some of us know our efforts are 'time limited' because of the realities of our profession's face-to-face work. Although there are highlights, it can sometimes feel like an unwinnable race to fill the gaps of other people's lives with band-aid solutions involving professionals, services, family and friends. In quieter moments, some of us reflect that service systems are failing our families not out of malice or intent, but from its constraints and outmoded values and methods. The social service industry in Australia was designed during the last century. In contemporary times, the industry neither reflects nor serves our families well. Despite calls for new knowledge which addresses some of the most complex issues facing our families, it is very rare investments are made in actions that catalyse innovation through cross-cultural and interdisciplinary exchange. This is why the First 1000 Days Australia 
model focuses on regional strategies that foster high levels of commitment to and alignment with the vision, values, resources and infrastructure to support family strengthening before during and after the first 1000 days.

When social work was taught in the late 1980s, our practice embodied the provision of support to families who needed help sorting out finances and getting hold of basics like beds and other furniture for their homes. Of course, the families and the need are still here, but social workers will more likely refer these families to home support services which themselves are limited in what they are able to provide due to recent significant budget cuts (Coggan, 2015). The needs of our families have increased in their complexity, the length of time families experience adverse stressors are much longer in their duration and the political landscape in Australia has not been able to adequately respond to calls from Indigenous peoples to ensure the human services workforce are culturally safe, culturally relevant and culturally competent. These factors, combined with the limitations of addressing Indigenous issues in the social work curriculum, do not prepare Indigenous social workers to influence the adoption and scale of interventions that work with our families, even though there is an evidence base which demonstrates lifelong benefits for children by supporting men and women during the early formation of their families (The Lancet, 2016).

\section{PREVENTION AS CHILD PROTECTION}

At the commencement of this century, Aboriginal ${ }^{1}$ people's experience of social work seems to be synonymous with child removal, with many claiming the profession is supporting another Stolen Generation (Smart, 2017). Indigenous social workers' decisions around child removal are simultaneously the worst part of and the one most rigorously considered. If a child needs to be removed in urgent circumstances, everything else stops until that child is safe. Removals can keep children out of harm's way, cause family's great grief and damage and cause distress to Indigenous social workers. Decisions in which removals fail families are those made when cultural safety is not understood or appreciated, the lived experiences of Indigenous peoples are not understood and the removals are done by workers invested in Euro-Western values, pedagogy and practice (Chong \& Arney, 2016). Contemporary child removals need to be

The term Aboriginal is used respectfully and is inclusive of Aboriginal and Torres Strait Islander peoples, Australia's two distinct Indigenous populations. 
understood in the context of history, where non-Indigenous social workers perpetuated the mass removals of Aboriginal children to placements in predominantly non-Aboriginal homes (Blackstock, Trocme \& Bennett, 2004).

In Australia, the rate of children in out-of-home care arrangements has doubled since the 2008 Apology to the Stolen Generation, ${ }^{2}$ causing many to refer to this distressing state of affairs as the second 'stolen generation' (Weston, 2018). In Victoria, as many as 32 per cent of Aboriginal children needing alternative care arrangements were not placed in accordance with the Child Placement Principle 3, which urges that Aboriginal children be placed with Aboriginal carers (Commissioner for Children and Young People, 2016). It is becoming an issue of national and international concern (Australian Institute of Health and Welfare, 2018), with leaders across community organisations and welfare groups calling for a coordinated, whole-of-community, whole-of-government approach to shift the service response from tertiary levelled interventions to prevention and early engagement with families. The Redfern Statement (National Congress of Australia's First Peoples, 2016), for example, emphasised the need for Aboriginal people to have a genuine say in their lives and the decisions that affect our peoples and communities. The Statement was backed by more than 50 organisations, a coalition that wants self-determining families, served by adequately funded agencies and calls for investments in First 1000 Days Australia (National Congress of Australia's First Peoples, 2016), a movement started by a Torres Strait Islander former social worker.

\section{CULTURE AND KNOWLEDGE: PATTERNED RESPONSES FOSTERING RESILIENCE AND PROTECTION}

First 1000 Days Australia ${ }^{3}$ aims to strengthen resilience, innovation and aspiration in Aboriginal and Torres Strait Islander families, as they grow their children to know who they are, where they come from and to whom they are connected. The model is informed by the understanding that culture is the protective factor for families and the importance of strong identity, belonging,

\footnotetext{
Between 1910 and 1970, many Aboriginal children were forcibly removed from their families as a result of various government policies. The generations of children removed under these policies became known as the Stolen Generations. These policies of child removal left a legacy of trauma and loss that continues to affect Aboriginal and Torres Strait Islander communities, families and individuals.See: https:// www.australianstogether.org.au/discover/australian-history/stolen-generations for more information. Accessed 29 April 2018.

See http://www.first1000daysaustralia.org.au/
} 
and fostering opportunities for people to transform their lives and have confidence in their ability to make change and theoretical concepts that link our human experience with that of 'country'. Social innovation is fostered through the co-development of effective solutions with families, in response to their aspirations and their ideas of social progress and indigenous innovation.

We know 'the world doesn't change one person at a time. It changes as networks of relationships form among people who discover they share a common cause and a vision of what's possible' (Wheatley $\&$ Frieze, 2006, p.1).

Our work fosters critical connections and joins together Indigenous worldviews with modern science and lived experiences. In these joint initiatives, we facilitate the process of emergence - allowing relationships, new knowledge and ways forward to emerge, rather than developing strategies, or implementation plans. This coming together is a patterned response, which strongly aligns with the nature-based principles of 'caring for country' or 'belonging to country' and other cultural determinants of health. This is because networks are the only form of organization on this planet used by living systems. These networks result from self-organisation, where individuals or species recognize their interdependence and organize in ways that support the diversity and viability of all.

Networks create the conditions for emergence, which is how life changes. Because networks are the first stage in emergence, it is essential we understand their dynamics and how they develop into communities then systems (Wheatley \& Frieze, 2006, p.1).

First 1000 Days Australia has always been positioned as a movement, rather than a program or a policy framework in which to work with families. We have not worried about critical mass, rather critical connections. We have not tried to convince large numbers of people to change; we have sought to connect with 'kindred spirits'; those like-minded others to develop the new knowledge, practices, courage and commitment that leads to broad-based change. As these networks developed into communities of practice through the establishment of working groups and event or purpose driven committees, we discovered that 
separate, local efforts connect as communities of practice and a new system suddenly and surprisingly emerged at a greater level of scale. What started in an Australian context has facilitated a community of practice focused on time specific, first nations' family empowerment activities into Indonesia and Norway. Taking social innovation (First 1000 Days Australia) to scale across regions, countries and networks of countries has demonstrated the power and influence of systems that emerge rather than those interventions which are only possible through planned, incremental change. (Wheatley \& Frieze, 2006, p.6).

\section{WHY FIRST 1000 DAYS AUSTRALIA?}

The internationally recognised 1,000 Days movement ${ }^{4}$ was established to improve maternal and infant nutrition, save lives and ensure that all children have the opportunity to reach their full potential. The movement combines evidence-based medical care and social support to address the United Nations' Sustainable Development Goals (UN Development Programme, 2016). The first 1000 days is now widely recognised as a period of 'maximum developmental plasticity and, therefore the period with the greatest potential to affect health and wellbeing over the life course' (Moore et al., 2017, p.1).

The Lancet 2016 series, 'Advancing early child development: From science to scale', identified 10 key messages of early childhood development, including the importance of the first 1000 days (The Lancet, 2016). It underlines neuro-scientific evidence that shows the association between low socio-economic status and poverty in early childhood and poor cognitive, academic and behavioural performance later in life. Poverty decreases carers' capacity to provide stimulating cognitive learning environments for their child, which makes them less likely to be able to provide the necessary learning environment for the child to gain a good start in life (Moore et al, 2017). Despite Australia's prosperity, early intervention supports for families and babies are not always available or accessible to those families experiencing hardship (Ritte et al., 2016, p.8). Even with a decade of the 'Close the Gap' campaign ${ }^{5}$ to redress poor health and wellbeing (Australian Human Rights Commission, 2018), too

\footnotetext{
See https://thousanddays.org/ for more information on the 1,000 Days movement internationally. The Campaign's goal is to close the health and life expectancy gap between Aboriginal and Torres Strait Islander people and non-Indigenous Australians within a generation. For more information go to: https://www.humanrights.gov.au/our-work/aboriginal-and-torres-strait-islander-social-justice/projects/ close-gap-indigenous-health. Accessed 18 February 2019.
} 
many Aboriginal and Torres Strait Islander children are living in complex situations or at heightened risk, and unable to live in sustainable, just and healthy communities (McLachlan, Gilfillan, \& Gordon, 2013).

At a time when more of our young are in need of protection (Council of Australian Governments, 2009), it is to Aboriginal and Torres Strait Islander people we should turn. Our communities intrinsically value children and are the experts when it comes to looking after them. Cultural knowledge held by Indigenous peoples continues to evolve and contribute to the raising of strong children across the world. Without a strong connection to culture or community, however, many young people do not understand the sacred responsibility of raising future Elders. Aboriginal and Torres Strait Islander communities hold knowledge of the important connection between people's health and wellbeing and the strength of culture, and of what works and what does not work in the raising of healthy and happy families (Sydenham, 2019).

A nutrition-focused program alone does not engage with the broader, holistic and cultural perspectives of Aboriginal and Torres Strait Islander people's health and wellbeing (National Aboriginal Community Controlled Health Organisation, 2006). The health and nutrition of men and women before conception is also important not only for pregnancy outcomes, but for the lifelong health of their children and the next generation (The Lancet, 2016). Recognising this, the University of Melbourne's Indigenous Health Equity Unit collaborated with Aboriginal organisations, community health groups, other research institutions, and government and international partners to broaden the nutrition focus of the international 1,000 Days movement and develop an Indigenous First 1000 Days Australia model. In creating this model, we needed a broader conceptual basis to redefine the first 1000 days interventions conceived of and led by Indigenous people.

\section{IMPLEMENTING SOLUTIONS USING A 'LIFECYCLE OF EMERGENCE FRAMEWORK'}

In considering the best theoretical models to underpin local and cultural solutions from pre-conception to a child's second birthday, the Lifecycle of Emergence Framework from the Berkana Institute provided the best process to facilitate the types of personal, professional and systemic transformations we were trying to achieve. Emergence is an inherently complex system construct 
that acknowledges the cycles of life, death and life. As such, it aligns most closely with the holistic concepts of health and wellbeing used by Aboriginal and Torres Strait Islander Australians:

\begin{abstract}
Aboriginal health means not just the physical well-being of an individual but refers to the social, emotional and cultural well-being of the whole Community in which each individual is able to achieve their full potential as a human being thereby bringing about the total well-being of their Community. It is a whole of life view and includes the cyclical concept of life-death-life (NACCHO, 2006).
\end{abstract}

The Emergence model starts with and acknowledges pioneers - those individuals, and institutions, who understand the need for change and are capable of taking steps to make it happen. The process of emergence then names these pioneering efforts in ways that others can identify with and facilitates pioneers to connect into networks of like-minded others. These networks are then nourished so they become cohesive, more integral and helpful to their members, and able to evolve into communities of practice that are self-organising and powerfully supportive within new networks.

First 1000 Days Australia is a facilitative process: we are pioneering new ways of thinking and acting, we train and support various workforces to make the transition from 'old' paradigm thinking to new processes that foster social innovation; and we develop methods and tools, group processes and practices to assist service delivery agencies and their workers commit to these new systems of open learning, which allows and celebrates family and community led engagement and to teach people how to implement cultural determinants of health and wellbeing (Pollard, 2010). These approaches are critical in a lifecycle of emergence because implementing solutions requires a focus on the underlying conditions that produce problems, rather than seeking solely to remedy the problems with which people present. Commonly called 'wicked problems' make it impossible to know the outcomes of our interventions beforehand (Brown, Harris \& Russell, 2010), which means we need to implement courses of action based on our best understanding of what will make a positive difference; namely, by starting with what families are doing well. Consequently, we develop strategies that support individuals and families to identify their aspirations, then assist them to meet these, rather than focusing on their deficits and needs. 
Because of this focus on aspirations not trauma, First 1000 Days Australia work needed a different conceptual model in which to work, one that begins with networks, shifts to intentional communities of practice and evolves into powerful systems capable of global influence (Wheatley \& Frieze, 2006). These dynamic processes mirror those found in living systems and are capable of extending beyond geographic, political, disciplinary and cultural boundaries, and, as such, are more readily adoptable and adaptable to our circumstances. In support of this, Wheatley and Frieze (2006, p.1-2) state:

Emergence is how life creates radical change and takes things to scale... By applying the lessons of living systems and working intentionally with emergence and its life cycle, we are demonstrating how local social innovations can be taken to scale and provide solutions to many of the world's most intractable issues.

Networks create the conditions for emergence and make it possible for people to find others engaged in similar work and take it to scale (Wheatley $\&$ Frieze, 2006). The Berkana Institute ${ }^{6}$ has developed a four-stage model to catalyse connections as the means of achieving global-level change: a strategy that names, connects, nourishes and illuminates collective thinking and action (Wheatley \& Frieze, 2006). The first stage identifies and emphasises pioneering efforts and names them as such. In the second, these pioneers are connected to others doing similar work to exchange ideas. The networks established by like-minded individuals are then nourished in the third stage, most essentially through creating opportunities for learning, sharing experiences and mobilising resources. This then facilitates a shift from individuals being part of networks into becoming communities of practice. The fourth stage illuminates these pioneering efforts through case studies and knowledge exchange processes, so that many more people learn from them.

\section{SUPPORTING A SYSTEM OF INFLUENCE}

The University of Melbourne undertook a nation-wide engagement process, hosting four national symposia. This was carried out to confirm the science of the importance of the first 1000 days in a child's life, and to determine

See http://berkana.org 
how an initiative focused on this time period could inform and improve current approaches to supporting families experiencing vulnerability. ${ }^{7}$ We linked Aboriginal and Torres Strait Islander families, Elders and representative organisations with scientific researchers, front-line workers (e.g. early learning educators, social workers, midwives and community workers), government policy makers, health economists and representatives from non-government organisations. The symposia revealed consensus for the importance of the first 1000 days, and the need to develop a model that communities would find appropriate and relevant.

Mapping the First 1000 Days Australia work against the Berkana Institute social emergence lifecycle, from the view of practitioners, shows how our work is both creating conditions for new ways to improve health and wellbeing outcomes during the period from pre-conception to the age of two, and developing systems of influence at a local and global scale. A commitment to the processes of emergence has facilitated us sharing our learnings with those confronted with change through structured presentations, short courses and workshops, and the reskilling of community people. First 1000 Days Australia has used this conceptual framework to support professionals and practitioners in their understanding of the impact of, and the need to change the use of, the deficit, targeted and racist language that permeates the service sector.

The emergence model has also supported the service delivery system's shift from a deficit to a strengths-based narrative of our families. We work with and across agencies in different regions to demonstrate how First 1000 Days Australia work can be used as a platform for joined up, cross-institutional and community action in regions. We also invest in media training for community people and support individuals and families, professionals and services to articulate the opportunities and experiences of those who have changed their language and their practice using our model. In doing so, we have identified and support a number of First 1000 Days Australia champions; those who recognise and mentor individuals in their community who are prepared to 'make the change', and demonstrate what is possible through innovation, being a champion and facilitating personal and systemic transformation.

The reports from these Symposia are available at: http://www.first1000daysaustralia.org.au/resources. 


\section{LIFECYCLE OF EMERGENCE THEORY: WHAT FIRST 1000 DAYS AUSTRALIA DOES}

First 1000 Days Australia creates pathways from old dominant systems into new systems which are geared toward supporting and actively encouraging our colleagues to change from outmoded ways of approaching early childhood development. By demonstrating the benefits of coming together, exchanging ideas, learnings and supporting each other through these transitions, we have been able to achieve policy and programming success.

\section{STAGE 1: CREATE CHANGE-CAPABLE NETWORKS}

The First Stage of Emergence is the creation of networks to promote societal change. Practitioners who use emergence as a theoretical basis need first to create coalitions, alliances and networks as the means for finding likeminded others to achieve change. First 1000 Days Australia aims for social transformation using an ecological framework to address the social and cultural determinants of health and wellbeing that affect Aboriginal and Torres Strait Islander peoples. In this phase, we identify change capable individuals, networks, and professionals by offering a series of information sessions and then, for those interested; offer a two-day short course to understand both the content and the opportunity of working within the First 1000 Days Australia model. After this introductory course; we then hold regional strategy meetings to identify a calendar of events for the year; and we establish a series of committees to progress work toward achieving what is incorporated in this calendar. A Regional Implementation Manager then provides support to a series of network meetings and task-oriented events. Through these and other activities, the first stage of emergence engaged members of the community in cultural and entrepreneurial activities, facilitated cross-agency meetings, local and regional governance structures were developed and media and communications training and support was provided to people in the region who were then prepared to become part of the First 1000 Days Australia Alumni and work together to create positive change for families. 


\section{STAGE 2: SUPPORT COMMUNITIES OF PRACTICE}

The second stage of emergence is the development of communities of practice made up of people invested in sharing common work. Emergence practitioners use relational communities to share what they know, support one another, and intentionally create new knowledge for their field of practice. They are communities of people who participate not only for their own needs, but to serve the needs of others. There is a shared understanding of success and a commitment to work towards achieving it.

First 1000 Days Australia generates important new knowledge that addresses some of the most complex issues facing our families and catalyses innovation through cross-cultural and interdisciplinary exchange. We invest in the development of communities of practice by embedding infant-centred practice into community leadership, agenda setting and decision making. We developed Charters and Principles that target the early years and support regional agencies to rebuild systems that are capable of responding to the aspirations of family members. The aim is to support all people engaged in this process to achieve and experience mastery. All effort is premised on the family remaining the primary and preferred site for developing and protecting culture and identity in Aboriginal children. During this phase, key cultural practices particularly ceremonial observances during the first 1000 days - are key investments, as are language and artisan skills. So too are the achievement of family goals, which include education, employment, financial management, nutritional advice and protective factors to support children to feel happy and safe.

\section{STAGE 3: HARNESS SYSTEMS OF INFLUENCE}

The third stage of emergence is the unpredictable sudden appearance of a system that has real power and influence. Pioneering efforts that hovered at the periphery suddenly become the norm, and practices developed by courageous communities become the accepted standard. People no longer hesitate about adopting these approaches and methods and learn them easily. Policy and funding debates include the perspectives and experiences of these pioneers, who are acknowledged as wisdom keepers for their particular issue. First 1000 Days Australia has had profound policy impacts - cited in national policy documents, funding strategies, national research funds and in organizational, local, regional and national programs. 
Our national impact has been because it is an evidence generative and evidence based initiative. The University of Melbourne has supported regions in their generation and use of evidence for impact. First 1000 Days Australia produces robust, applicable evidence about what works, promotes the implementation of high-impact and cost-effective programs, and enables our ability to influence the adoption and scale of such interventions. We undertake workforce development, train peer researchers, do community-led research and employ Indigenous methods of knowledge generation.

Our emerging system of influence is evident through local and regional programming, the creation of cultural programs specific to First 1000 Days Australia activities, policy production processes across all levels of government, and in research strategy and implementation. This new system of influence is focused on family aspirations and is family led, clearly an alternative to the current dominant support system that is typically hierarchical, racialised and oppressive. The current system has also been incapable of responding to Australian Aboriginal people as equal citizens with leadership skills, cultural practices and life ways that are appropriate for the entire nation (Arabena, 2018). When alternative systems of emergence start to appear, dominant systems will want to preserve themselves as they begin to decline. While First 1000 Days Australia is driving a new emerging system, it has compassion for those invested in the dominant system, which is not meeting the needs or expectations of the people they are meant to serve.

\section{PATHWAY OF CHANGE: EVIDENCE AND TRANSFORMATION}

Social work practice is based on a rights agenda in which all people have the right and responsibility to reach their full potential. The premise underlying First 1000 Days Australia is that the role of protecting children is best undertaken by a child's family - a multigenerational, non-biological and diverse model of family that includes mothers, fathers and/or care givers, grandparents and other relatives and friends. First 1000 Days Australia's strengths-based approach to working with families supports the development of family histories and cultural identity, facilitates access to Country and kin, focuses on the roles and responsibilities of fathers and mothers, encourages the use of therapeutic cultural supports, undertakes multigenerational family studies and promotes family entrepreneurship. 
We use and explore Indigenous concepts of parenting to address antenatal engagement through whole-of-family, whole-of-service approaches that incorporate life skills, early learning and education to build resilient families. We emphasise the rights of children yet to be conceived (First 1000 Days Australia Council, 2017) to be born into families who have loving expectations of them, are hopeful about their future and will help them to achieve their aspirations. We are supporting families in their gaining of information on how to choose to become parents, to participate in their child's learning, and to access antenatal and early years support informed by cultural parenting that honours the role of both men and women in nurturing children. First 1000 Days Australia works with community leaders to move away from family dependence on welfare, to maximise protective factors and to support the achievement of life goals by using household planning, life coaching and mentoring. There are multiple pathways that can be used to transform the circumstances of families who experience vulnerability, many of them conceived by the families themselves.

\section{CREATING NEW KNOWLEDGE}

Terms such as evidence based or evidence informed do not fully cover the evidence-generative capabilities of First 1000 Days Australia initiatives. Evidence generated through university processes is predominantly considered to be privileged, excluding First People's knowledge systems and denying the validity of how Indigenous knowledge is generated and communicated. Emergence theory as an underpinning conceptual framework in a research-driven strategy is not without its challenges. Researchers are learning what is required to fit their research activities into an Indigenous knowledge production framework, and Indigenous peoples are learning to trust research after having been for decades the 'most researched people on the planet' (NACCHO, 2006).

To ensure that First 1000 Days Australia has a positive impact and builds a robust evidence base for the future, a systematic research program is being undertaken to generate, link and use data from across our active sites based on four overarching research objectives:

1. To understand and quantify the characteristics that lead to thriving, strong and resilient Aboriginal and Torres Strait Islander families. 
2. To identify the key determinants of environmental, cultural, familial, newborn and child health, and the predictors of health and wellbeing outcomes at two years of age and again at school entry.

3. To evaluate and review health and wellbeing strategies implemented through First 1000 Days Australia to ensure their alignment with the needs of families and community in real time.

4. To develop an Indigenous-led research infrastructure legacy, upon which future Aboriginal and Torres Strait Islander research and researchers can build.

To facilitate research translation on this and other work from across Australia and the world. Our research to date can be found on our website, which is a catch all for social media, training courses, presentations, videos and cultural activities.

\section{CASE STUDIES OF 'SOCIAL EMERGENCE' IN FIRST 1000 DAYS AUSTRALIA}

The following case studies are examples of how we are implementing First 1000 Days Australia's regional activities, all of which follow similar preliminary engagement patterns. Our staff, either from the University of Melbourne or other agencies, Indigenous and expert content specialists, are often times invited into regions to deliver a series of workshops and, if there is interest, follow up with short courses and regional implementation workshops, and support the establishment of working groups to progress key First 1000 Days Australia activities. Some working groups engage Aboriginal and Torres Strait Islander dads, provide cultural supports through Welcome Baby to Country events, or guide the undertaking of household-level surveys to understand aspirations in family groups and households.

\section{STAGE 1: NETWORKS OF PIONEERS}

More than a Landlord, Aboriginal Housing Victoria, Northern Melbourne Recognising that the provision of safe, stable and affordable housing is the first step in building pathways to improved lives, Aboriginal Housing Victoria (AHV) developed the More than a Landlord (MTAL) program in partnership with First 1000 Days Australia. MTAL evolved from a program of 
nutritional activities to a holistic life-coaching service informed by a household-level survey. This survey was developed with AHV tenant families to capture their aspirations and future needs, which were then fed back to AHV. This information led AHV to employ life coaches to engage with tenants to make plans based on their aspirations, and then to connect them with appropriate services and local programs that aligned with these goals. MTAL, which is strengths-based and focused on empowerment and self-determination, not only captured important data to inform and influence AHV housing policy decisions and service responses. It also enabled AHV to support the social and emotional wellbeing outcomes of their tenants in a holistic manner by supporting their aspirations rather than by simply dealing with their problems.

\section{Peer research for job readiness}

Peer researchers are the primary conduit for engaging families with activities carried out by both First 1000 Days Australia and the regional lead agency. Employed by the lead agency and recruited from among families residing within the regional area, peer researchers play a key role in supporting access to, engagement with and the reach of First 1000 Days Australia into the community. Peer researchers are crucial to the development of the household survey, and are trained in the survey protocol and contents, informed consent process, survey methods, survey technologies and techniques for visiting households safely. Once trained, their relationship with service providers shifts, as they are no longer interacting with agencies within the client discourse, but became active advocates for families in their community and colleagues to other community leaders. They are also engaged with Aboriginal services as decision makers, employees, sole traders and consultants. For example, two of the five peer researchers from AHV went on to be offered full-time work at AHV, one was commissioned to do artwork, and three were pursuing further education at high school or studying for a Diploma in Community Services.

\section{STAGE 2: NOURISHING THE NETWORKS}

Australian Indonesian Collaboration - Finding patterns across Indigenous populations in Australia and Indonesia

The First 1000 Days Australia team reached out to Indonesian universities in 2016 to establish connections with leading academics who were progressing similar early childhood aims: specifically, exploring strategies to prevent 
non-communicable diseases (NCDs) during pregnancy and early infancy in Indonesian Indigenous communities. In both countries, the 1,000 Days movement was emerging in a variety of ways across political domains and in grassroots programs. A core project group of academics from Indonesia and Australia was established as part of larger health initiative led by the Australian-Indonesia Centre to prevent NCDs. In both countries, the project team contacted their wider networks and brought together stakeholders from services, policy, academia, non-government organisations and front-line workers in two roundtable discussions. Despite language barriers and diverse cultural and historical differences, people were able to discuss innovative concepts, approaches and learnings and raise important questions. Participants found common ground in the importance of holistic approaches to infant and child health. They agreed that the health and wellbeing of a child benefits from a foundation in pre-conception health and family planning with parents. Participants also understood the importance of policy and multisectoral coordination and collaboration.

The roundtable discussions inspired conversation and pledges for action in much wider circles, and motivated a number of Indonesian organisations, individuals and students to contact First 1000 Days Australia with requests to undertake further research together. Implementation and research projects, based on the expanded Australian model of the First 1000 Days, have now commenced in Indonesia. The Indonesian Australian collaboration of multisector partners demonstrates the impact of a movement that can emerge globally but embed itself locally in different forms, according to different community contexts.

Norway - Using Indigenous methodologies to strengthen cultural connection, language use and identity with Sámi in Norway

First 1000 Days Australia's Indigenous-led engagement methods (Ritte et al., 2016) were used to develop a series of workshops, focus groups and collaborative visits at the University of Melbourne and at the University of Tromsø in Norway between researchers, social workers and front-line workers. This network has led to the genesis of a First 1000 Days model that is applicable to Sámi families in Norway. Our initial activities in Norway incorporate Sámi community traditions, including an emphasis on extended family. Sámi grandparents, for example, are being invited, alongside professionals, to teach 
Sámi languages to parents and soon-to-be parents. As such, the workshops are encouraging parents and grandparents to take on a strong role in the teaching of Sámi languages and culture to their children. As with our work in Australia, there is a strong emphasis on engaging men during the early formation of their families. A Sámi Social Worker is leading the effort across the Arctic Circle and First 1000 days work is funded by the Sámi Parliament. In 2019, the Year of Indigenous Languages, the Sámi First 1000 Days project will present their work on the 'Father Tongue' supporting men engaging with Sámi language and sharing what they learn with their babies and partners, prior to the child being born.

\section{STAGE 3: SYSTEMS OF INFLUENCE - WHERE NEW PRACTICES BECOME THE NORM}

\section{Entrepreneurial action: Growing Indigenous business}

First 1000 Days Australia facilitated a two-day short course in Townsville, a city on the north-east coast of Queensland, Australia, where Aboriginal and Torres Strait Islander participants identified keen interest in business development and entrepreneurial ventures within their community. Some participants shared a business concept but were unsure how to progress beyond concept phase. They were motivated to seek business start-up and developmental support from a Queensland Government Department of Aboriginal and Torres Strait Islander Partnerships representative, who provided additional support and information on how to start a business. First 1000 Days Australia is partnering with the Department to respond to these expressions of interest, by providing assistance and support in planning, coordinating and delivering workshops prior to Indigenous Business month, which is held in October every year. This partnership draws on the expertise of local businesses, consultants and the wider business community to ensure that local people receive the relevant support and assistance they need to expand their capacity to bring new business models to fruition. A 'IndigIdeas' event was held, in which four Indigenous businesses had the opportunity to present to a panel for a prize pool that included cash, access to mentoring and business advice. IndigIdeas is now being trademarked for licensing around Australia to support Indigenous start ups and will be expanded to operate nationally. 
First 1000 Days Moreton Bay - Shifting from family crisis to family aspirations

A cross-organisational and trans-disciplinary executive group of local Elders, dieticians and representatives from early childhood services regularly meet to coordinate the development of First 1000 Days Australia initiatives with Aboriginal and Torres Strait Islander families living in the region. These have, so far, included BBQs and yarning circles, a Welcome Baby to Country ceremony during NAIDOC week, ${ }^{8}$ and a month of workshops to develop family entrepreneurial activities. The development of a household survey of local Aboriginal and Torres Strait Islander families led to the engagement of fourteen peer researchers with First 1000 Days Australia research activities, and they brought new ideas on how to reach and engage families and how to articulate and respond to their aspirations. Momentum in the area is growing, with both local organisations and the Queensland State Government offering support by providing funding for workshop rooms or resources for activities. These included resources for Elders to build a coolamon (an Aboriginal carrying vessel), create Gubbi Gubbi headpieces for infants, and to source kangaroo skins to give to babies at the Welcome Baby to Country ceremony. Local demand for the ceremony in the Moreton Bay area was so great that a second was held on Aboriginal and Torres Strait Islander Children's Day in August. As a result of this work, positive media stories were reported and a partnership resource was developed to support communities around Australia facilitate Welcome Baby To Country Ceremonies ${ }^{9}$.

\section{STAGE 4:ILLUMINATING FOR SUCCESS}

It is difficult for anyone to see work based on a different paradigm. If people have noticed the work of First 1000 Days Australia, it is often characterized as an inspirational deviation from normal practice. It takes time and attention for people to see different approaches for what they are: examples of what the new world could be. First 1000 Days Australia has a website with all our publications, our training programs and conference presentations. We hold summits, host learning journeys and have built a program of activity on

8 NAIDOC (National Aborigines and Islanders Day Observance Committee) Week is held across Australia each July to celebrate the history, culture and achievements of Aboriginal and Torres Strait Islander peoples. For more information go to: https://www.naidoc.org.au/about/naidoc-week.

9 The Welcome Baby to Country Resource Brochure is available from the First 1000 Days Australia website: http://www.first1000daysaustralia.org.au/sites/default/files/report_pdf/FTDA_WelcomeBaby_ guidelines_FINAL.pdf 
and with pioneers. First 1000 Days Australia work has been shared in local, regional, national and international forums; through social media and at community meetings. We facilitate culture led work; pioneer work with men and we develop resources on behalf of families and communities.

\section{WHAT WE HAVE LEARNED: ESSENTIALS FOR SUCCESS}

Emergence is an inherently complex-system construct. Rather than trying to create communities, what this model does is acknowledge pioneering efforts, then name them in ways that others can identify with, thereby allowing these pioneers to coalesce and connect. The First 1000 Days Australia model uses an ecological framework of social and family-based strategies and participatory research methods to provide a comprehensive, rigorous and consistent empirical evidence base. As an Indigenous-led, holistic initiative with interventions designed and implemented under the direction of community, our goal is to ensure that knowledge is returned to the community. Using social emergence as a principle and goal, we are engaging with young people and families and providing them with opportunities to strengthen their extended family and community networks according to their own aspirations.

The engagement of health care workers, community organisations and all levels of government is essential to address local and systemic-level issues contributing to the poor infant and family health in many of our communities. Additional care and support is needed to encourage service providers to act on evidence, to build their service and regional level capacity to respond to the aspirations of families, and to change their language from deficit discourse to strengths-based narrative. Regional social emergence implementers need to focus on population-level place-based approaches, capacity building and knowledge exchange, to utilise political and advocacy experience, and to support family-led solutions and enterprise as a strategy to shift welfarist thinking to deliberate and affirmative action.

In an Indigenous context, community governance is an essential ingredient of successful social emergence work, particularly when it is committed to developing networks and communities of practice informed by research, innovation and interventions. First 1000 Days Australia initiatives are led by Aboriginal regional implementation managers based at each lead agency. These managers have a key role in facilitating social emergence and work 
predominantly to create networks that value the community as co-designers, co-implementers and co-knowledge translators of research and outcomes.

Lead agencies working towards establishing First 1000 Days Australia in their region are supported to build the capacity of staff and undertake a needs assessment to work out a program logic for developing and implementing First 1000 Days Australia strategies. Local governance processes determine the exact nature of appropriate, community-specific interventions and tailors capacity-building strategies to the aspirations of their community. In this way, Aboriginal and Torres Strait Islander families are empowered to take control of their destiny through identifying their own aspirations and strengths, and through having agency over the solutions they can utilise to meet them.

The First 1000 Days Australia model will continue to evolve with the ongoing engagement of families and the wider community in the emergence of a new system of influence. Aboriginal and Torres Strait Islander communities have been nurturing and raising strong children in culture for millennia. We are sharing the story of parenting with other First Nations people in Indonesia and Norway. We are building an evidence base informed by this culture, from which all families can benefit and give their children the best start in life.

\section{REFERENCES}

Arabena, K. (2018). Welcoming the Treaty Generation: The First 1000 Days. In Griffith Review 60: First Things First (https://griffithreview.com/articles/ preparing-treaty-generation-first-1000-days/). Brisbane: Griffith University. Australian Human Rights Commission. (2018). Close the Gap: Indigenous Health Campaign. Australian Human Rights Commission. Retrieved from: https:// www.humanrights.gov.au/our-work/aboriginal-and-torres-strait-islandersocial-justice/projects/close-gap-indigenous-health

Australian Institute of Health and Welfare. (2018). Child Protection Australia 2016-17. Canberra: Australian Government.

Blackstock, C., Trocme, N., \& Bennett, M. (2004). Child Maltreatment Investigations among Aboriginal and non-Aboriginal Families in Canada. Violence against Women, 10(8), 1-16. https://doi.org/10.1177/1077801204266312

Brown, V., Harris, A. J., \& Russell, Y. J. (2010). Tackling Wicked Problems: Through the transdisciplinary imagination. London and Washington DC: Earthscan. https://doi.org/10.4324/9781849776530 
Chong, A., \& Arney, F. (2016). Australia failing to safeguard cultural connection for Aboriginal children in out of home care. The Conversation. Retrieved April 29, 2018, from: http://theconversation.com/australia-failing-to-safeguardcultural-connections-for-aboriginal-children-in-out-of-home-care-68051.

Coggan, M. (2015). Budget 2014: $\$ 534$ million cut to Indigenous programs. $A B C$ Nerws. Retrieved April 28, 2018, from: http://www.abc.net.au/news/2014-0513/budget-2014:-\$534-cut-to-indigenous-programs-and-health/5451144.

Commissioner for Children and Young People. (2016). Always was, always will be Koori Children: Systemic inquiry into services provided to Aboriginal children and young people in out of home care in Victoria. Melbourne: Commissioner for Children and Young People.

Council of Australian Governments. (2009). Retrieved from Protecting Children is Everyone's Business: National Framework for Protecting Australia's Children 2009-2020. Retrieved from: https://www.dss.gov.au/sites/default/files/ documents/child_protection_framework.pdf

First 1000 Days Australia Council. (2017). The Charter of rights for children yet to be conceived. Melbourne: First 1000 Days Australia. Retrieved November 21, 2018 from: http://www.first1000daysaustralia.org.au/charter-rights-childrenyet-be-conceived.

McLachlan, R., Gilfillan, G., \& Gordon, J. (2013). Deep and Persistent

Disadvantage in Australia. Retrieved from Australian Government Productivity Commission: http://library.bsl.org.au/jspui/bitstream/1/3521/1/Deep\%20 and\%20Persistent\%20Disadvantage\%20in\%20Australia_PC\%20July2013.pdf. Moore, T. G., Arefadib, N., Deery, A., \&West, S. (2017). The First Thousand Days: An Evidence Paper - Summary. Parkville, Victoria: Centre for Community Child Health and Murdoch Children's Research Institute.

National Aboriginal Community Controlled Health Organisation. (2006).

Definitions. Retrieved from: http://www.naccho.org.au/about/aboriginalhealth/definitions.

National Congress of Australia's First Peoples. (2016). The Redfern

Statement. Retrieved from: https://nationalcongress.com.au/wp-content/ uploads/2017/02/The-Redfern-Statement-9-June-_Final.pdf

Pollard, A. (2010). Professionalism and pedagogy: A contemporary opportunity. London. Ritte, R., Panozzo, S., Johnston, L., Agerholm, J., Siv, K., Rowley, K., \& Arabena, K. (2016). An Australian Model of the First 1000 Days: An Indigenous-led process to turn an international initiative into an early life strategy benefiting Indigenous families. Global Health, Epidemiology and Genomics, 1(1). https://doi.org/10.1017/gheg.2016.7 
Salmon, M., Doery, K., Dance, P., Chapman, J., Gilbert, R., Williams R., \& Lovett, R. (2018). Defining the Indefinable: Descriptors of Aboriginal and Torres Strait Islander Peoples' Cultures and their Links to Health and Wellbeing. Aboriginal and Torres Strait Islander Health Team, Research School of Population Health, Australian National University: Canberra, http://dx.doi. org/10.25911/5bdbcdf5c89a7

Smart, J. (2017). Child Protection and Aboriginal and Torres Strait Islander Children - Child Family Community Australia Resource Sheet, August 2017. Australian Institute of Family Studies. Retrieved April 28, 2018, from: https://aifs.gov.au/ cfca/publications/child-protection-and-aboriginal-and-torres-strait-islanderchildren

Smart, J. (2017). Risk and Protective Factors for Child Abuse and Neglect - Child Family Community Australia Resource Sheet. Australian Institute of Family Studies. Retrieved from: https://aifs.gov.au/cfca/publications/risk-andprotective-factors-child-abuse-and-neglect.

Sydenham, E. (2019). Ensuring Equality for Aboriginal and Torres Strait Islander Children in the Early Years. A Discussion Paper. Melbourne: Early Childhood Australia and SNAICC (Secretariat of National Aboriginal and Islander Child Care). Retrieved February 18, 2019 from: http://www. earlychildhoodaustralia.org.au/wp-content/uploads/2019/02/SNAICC-ECADiscussion-Paper-.pdf.

The Lancet. (2016). Advancing Early Childhood Development: From science to scale. An executive summary for The Lancet's Series. The Lancet.

United Nations Development Programme. (2016). Sustainable Development Goals. Geneva: UNDP. Retrieved April 28, 2018, from: http:/www.undp.org/ content/undp/en/home/sustainable-development-goals.html.

Weston, R. (2018). Indigenous children in care doubled since stolen generations apology. The Guardian. Retrieved from: https:/www.theguardian.com/ australia-news/2018/jan/25/indigenous-children-in-care-doubled-sincestolen-generations-apology.

Wheatley, M., \& Frieze, D. (2006). Margaret Wheatley Writings: Using emergence to take social innovation to scale. Retrieved April 28, 2018, from: http://www. margaretwheatley.com/articles/emergence.html. 